\title{
Investigation of the friction induced conformational change of protein and wear of UHMWPE by a wear process with microfabricated surfaces
}

\author{
Hsu-Wei Fang a,*, Yu-Chih Su ${ }^{\text {a }}$, Huei-Ting Huang a , Wei-Bor Tsai ${ }^{\mathrm{b}}$, Hsuan-Liang Liu ${ }^{\mathrm{a}}$ \\ a Department of Chemical Engineering and Biotechnology E Institute of Biotechnology, National Taipei University of Technology, Taipei, Taiwan \\ ${ }^{\mathrm{b}}$ Department of Chemical Engineering, National Taiwan University, Taipei, Taiwan
}

\section{A R T I C L E I N F O}

\section{Article history:}

Received 3 August 2007

Received in revised form 13 September 2008

Accepted 22 September 2008

Available online $\mathrm{xxxx}$

\section{Keywords:}

Polyethylene (UHMWPE)

Wear

Albumin

Protein conformation

\begin{abstract}
A B S T R A C T
An accelerated wear testing procedure was developed to carry out an articulation process between UHWMPE and microfabricated surfaces with controlled asperities. By such a design, the effect of lubricant on wear of UHMWPE can be scaled-up and measured within a short duration of the test. The most abundant composition of the synovial fluid-human serum albumin was employed as a lubricant. Analysis of the albumin protein structure by a circular dichroism (CD) spectroscopy was proceeded to detect the conformational change during a tribological process. We observed that the thinner and fibril-like wear particles were found in biological lubricants than the particle generated in water. Our results also indicated that the content of $\alpha$-helix structure of albumin was decreased after the tribological process. The denatured albumin solution resulted in the decrease of UHMWPE wear rate. It may be due to the decrease of the contact angle of unfolding albumin protein on articulating surfaces. It implies a larger coverage of the lubricating molecules on the UHMWPE surface. The relevance of the tribological process induced conformational change and wear of UHWMPE were discussed in this study.
\end{abstract}

(c) 2008 Elsevier B.V. All rights reserved.

\section{Introduction}

Total joint replacements have been successfully implanted clinically to replace damaged human joints for several decades [1]. A total joint replacement system comprises of a pair of material surfaces articulating to each other to simulate the functions of the human joints. From years of researches and developments, an artificial joint system consisting of $\mathrm{Co}-\mathrm{Cr}-\mathrm{Mo}$ alloy sliding on an ultra-high molecular weight polyethylene (UHMWPE) surface has been proven to function well and widely used clinically. Earlier problems of the total joint system include the fracture and fixation problem of the joint implant. Optimizing of the material strength and minimizing its difference from the human bones have resolved most of the fracture problems $[2,3]$. The introduction of bone compatible porous surface coatings on the stem to provide another choice for fixation has helped to almost eliminate the fixation problem [4-6]. Currently, wear of UHMWPE and wear particles induced osteolysis and bone resorption are the major factors causing the failure of the total joint replacement. In the past several years, wear of UHMWPE has been dramatically reduced by using highly cross-linked UHMWPE [7-9]. However, these still exists feasibility to further improve the lubrication and to reduce

\footnotetext{
* Corresponding author. Department of Chemical Engineering, Mail stop 2521, National Taipei University of Technology, 1, Sec. 3, Chung-Hsiao E. Road, Taipei 106 Taiwan. Tel.: +886 930380200; fax: +886 227418575.

E-mail address: hwfang@ntut.edu.tw (H.-W. Fang).
}

the wear of the artificial joints in-vivo. We need further understanding of the lubrication mechanism of synovial fluid.

On the other hand, recent interest in minimally invasive techniques has made partial joint replacement such as unicompartmental knee arthroplasty (UKA) regained the popularity. Short-term results with respect to pain relief and function are equal to or better than those of total knee replacement. However, with longer-term follow-up, the failure rate is higher than that of total knee replacement. The most common mechanism of failure includes component loosening, polyethylene wear and progression of disease in the un-resurfaced compartment. Degeneration in the opposite compartment is a common cause of failure after unicompartmental arthroplasty [10,11]. Elsaid et al. [12] indicated that poor lubrication may be related to the cartilage damage. Under UKA design, the influence of conformational changed fluids from the joint implant interface on the lubricating ability of the natural cartilage on the other side may be critical. If the association of the tribochemistry of biological lubricants due to the joint implant articulation and the osteoarthristis of the cartilage could be identified, further development shall be made to enhance the durability of UKA.

Further measurements and quantification of the tribochemical reactions of synovial fluid and its compositions under the articulation of artificial joints are necessary. From an engineering viewpoint, synovial fluid can be viewed as a lubricant containing albumin proteins, globulin proteins, lipids, hyaluronic acid, etc. Among which, $56 \%$ are albumin. It was also indicated that the albumin contents in the synovial fluid decreased to $42 \%$ in rheumatoid arthritis patients [13]. 


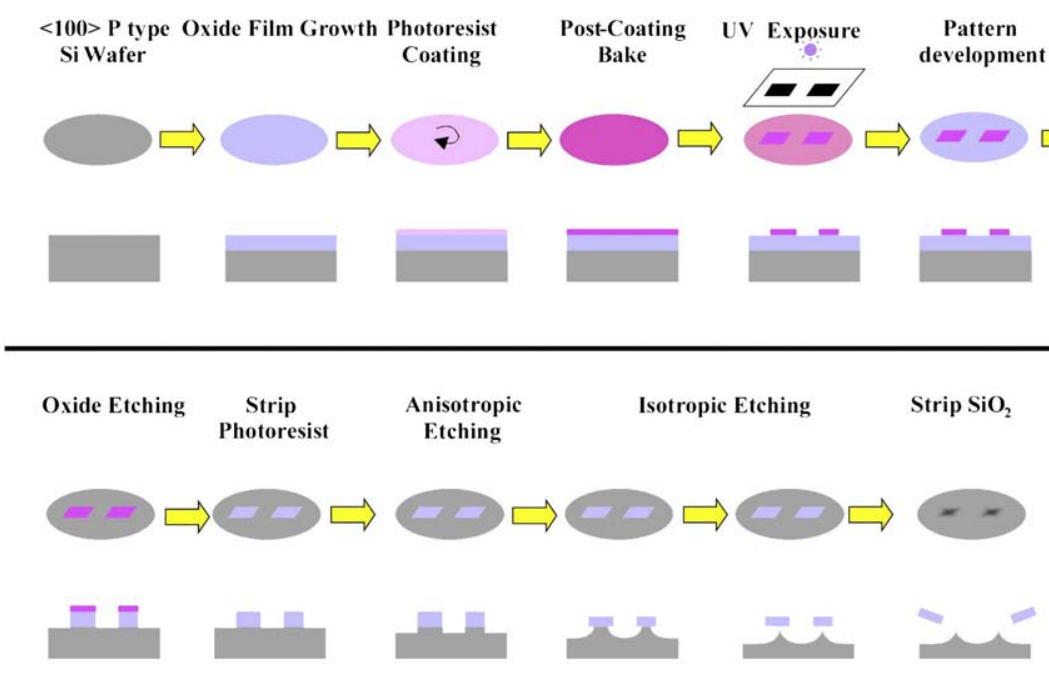

Fig. 1. Flow chart of the microfabrication process of surface textures on the silicon.

We need to further understand what happens to the most abundant protein in synovial fluid-albumin under a tribological process of the artificial joint materials. Previously, Fang et al. [14-16] have successfully applied surface texturing techniques to prepare narrowlydistributed UHMWPE particles with controlled sizes and shapes. This accomplishment enables the bioactivity evaluation of different sizes and shapes of UHMWPE wear particles $[17,18]$. The influence of biological lubricant on the morphology of UHMWPE wear particles with surface textures have been reported [19]. In this study, we further developed an accelerated wear testing procedure by rubbing the microfabricated silicon surface textures with UHMWPE. By such a design, the effect of lubricant on wear of UHMWPE can be scaled-up and measured within a short duration of the test. The lubrication performance can also be evaluated by wear rate and the morphology of the generated wear particles. Human serum albumin was employed as a lubricant. The association of albumin concentration, conformation change, and wear of UHMWPE were investigated and analyzed.

\section{Materials and methods}

An accelerated wear testing procedure was developed to perform the experiments. Surface textures with controlled asperities of the silicon wafers were prepared by a microfabrication process. Analysis of the albumin protein structure was carried out by circular dichroism (CD) spectroscopy. The details of the materials and experimental procedures are described below:

\subsection{Materials}

Raw GUR1050 UHMWPE materials obtained from United Orthopaedic Corporation, Taiwan were used in this study. UHMWPE cylinder pins were machined to $6.35 \mathrm{~mm}$ in diameter and $25.4 \mathrm{~mm}$ in length with diamond turning on both end surfaces without polishing. The mean roughness ( $\mathrm{Ra}$ ) of UHMWPE pins' end surface is $0.82 \mu \mathrm{m}$. Human serum albumin (Sigma, AG-1653) solutions with three different concentrations $(6.3 \mathrm{mg} / \mathrm{ml}, 12.6 \mathrm{mg} / \mathrm{ml}, 25.2 \mathrm{mg} / \mathrm{ml}$ ) were used as lubricants in the experiments. All UHMWPE pins were presoaked in the albumin solution for at least 15 days so as to become completely saturation.

\subsection{Microfabricated surface textures}

The silicon wafer surface with controlled asperities was prepared by photolithography patterning and etching of the bulk substrate. Fig. 1 shows the flow chart of the microfabrication process. Two-inch diameter polished type P silicon wafers with (100) orientation purchased from Summit-Tech were used as the substrate material. Wet oxidation of the silicon wafers was carried out in a glass-tube oven at $1100{ }^{\circ} \mathrm{C}$ for 135 min to form a silicon dioxide film with a thickness of $1 \mu \mathrm{m}$. A pattern of rectangles with different size and aspect ratios were made on a chrome direct-writing photomask. The silicon dioxide surface was spin coated with a Shipley 1813 positive photoresist. The dark-featured photomask was then placed on the photoresist surface and exposed to a UV source in a mask aligner to decompose the surrounding polymer surface leaving a positive rectangular pattern. The decomposed photoresist polymer was then removed in a Shipley 351 developer. The resulting positive photoresist surface was then etched first with a BOE solution (diluted buffer HF solution) to etch away the $\mathrm{SiO}_{2}$ in a wet chemical bath. The photoresist was then removed by washing with acetone-alcohol. Subsequently, the silicon material was subjected to isotropic silicon etching (HNA etchant; liquid volume ratio $\mathrm{HF}: \mathrm{HNO}_{3}: \mathrm{CH}_{3} \mathrm{COOH}=8: 75: 17$ ) in a wet chemical bath at room temperature. The $\mathrm{SiO}_{2}$ layer was removed after an isotropic undercutting etching process. The resulting surface features are an array of rectangular ridges with sharp edges. Finally,

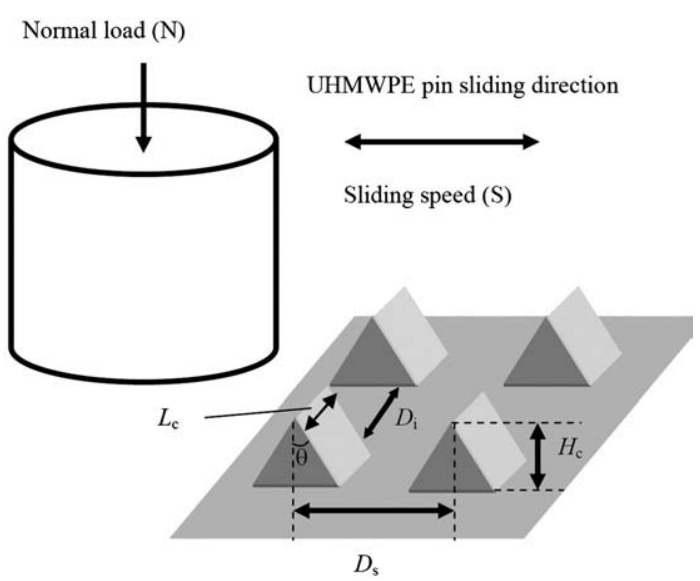

$L_{\mathrm{c}}$ : cutting-edge length

$H_{\mathrm{c}}$ : cutting-edge height

$W_{\text {c }}$ : cutting-edge width

$D_{\mathrm{s}}$ : pitch in the sliding direction

$D_{\mathrm{i}}$ : distance between adjacent features

Fig. 2. UHMWPE particle generation by a micro-cutting process. 
(a)

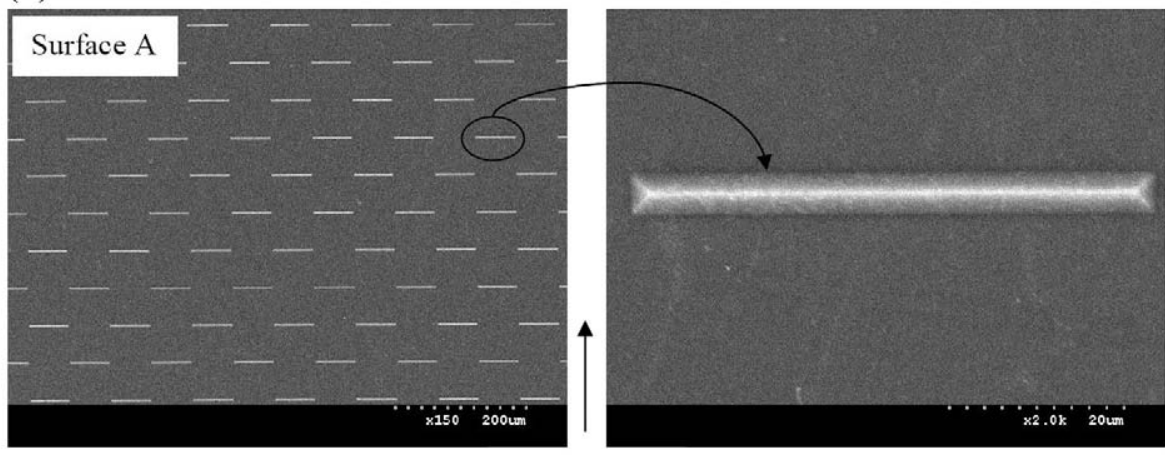

(b)

Sliding direction

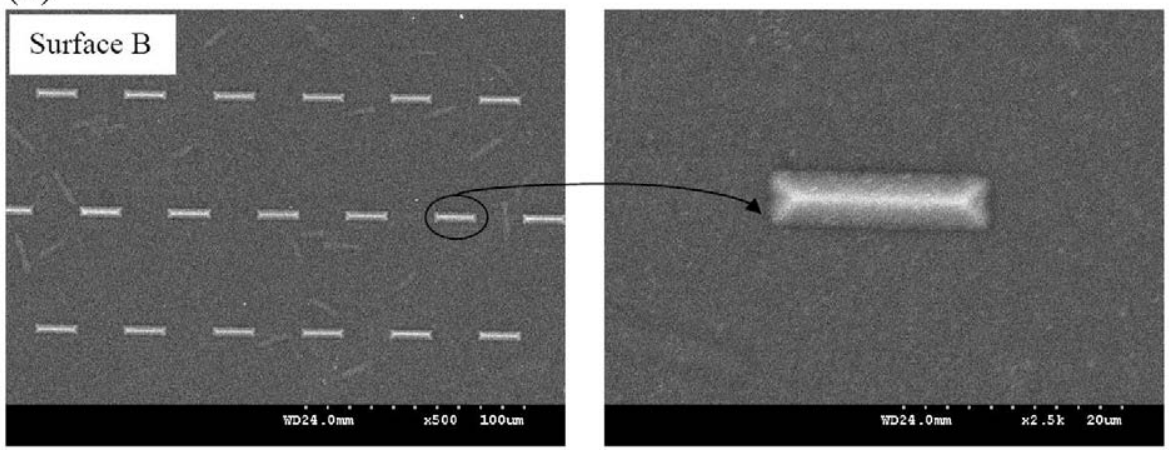

Fig. 3. SEM images of microfabricated surface textures.

a layer of $5 \mathrm{~nm} \mathrm{Cr}$ coating was evaporated onto the surface to increase the wear resistance of the surface. The heights of the surface textures were measured by a Mahr profilometer and the feature lengths and widths were measured from SEM observations.

\subsection{Wear tests}

The principles of surface-texture design to generate UHMWPE wear particles have been developed previously [10]. By rubbing UHMWPE with the cutting edges of the silicon surface textures, the UHMWPE wear particles were generated. A schematic diagram of this micro-cutting process and the critical dimensions of the surface textures are shown in Fig. 2. A linear reciprocating wear test was applied to generate UHMWPE wear particles. ASTM F732 was used as a guideline [20]. One UHMWPE pin and three control pins were weighed three times. Three control pins were soaked in the lubricant at the same liquid level as the wear-testing condition. The UHMWPE pin was then mounted on the tester. Linear reciprocating wear tests were run under a nominal contact pressure of $3 \mathrm{MPa}$, a stroke length of $19 \mathrm{~mm}$, a frequency of $1.5 \mathrm{~Hz}$, and an average sliding speed of $57.2 \mathrm{~mm} /$ s. After the wear test, the four pins were weighed and the wear loss of the testing pin was obtained after adjusting the weight change from the control pins. The wear tests were repeated 3 times.

\subsection{Wear particle characterization}

UHMWPE particles were collected by repeated water rinsing of the sample, sample holder, and parts that came into contact with

Table 1

Surface-feature dimensions of microfabricated surface textures

\begin{tabular}{lll}
\hline & Surface A & Surface B \\
\hline Feature length & $56.0 \mu \mathrm{m}$ & $15.6 \mu \mathrm{m}$ \\
Feature width & $4.6 \mu \mathrm{m}$ & $4.8 \mu \mathrm{m}$ \\
Feature height & $2.1 \mu \mathrm{m}$ & $2.0 \mu \mathrm{m}$ \\
Distance between features (in sliding direction) & $100 \mu \mathrm{m}$ & $100 \mu \mathrm{m}$ \\
\hline
\end{tabular}

particles, into a sterilized beaker. In order to digest the proteins in the albumin solutions, $5 \mathrm{~mL}$ collected solution was added with $20 \mathrm{~mL}$ $\mathrm{NaOH}$ solution $(5 \mathrm{~N})$ in a $65{ }^{\circ} \mathrm{C}$ water bath for $24 \mathrm{~h} .3 \mathrm{~mL}$ digested solutions were added with $2 \mathrm{~mL}$ sucrose gradient solutions $(5 \%, 10 \%$, $20 \%$, and 50\%). The solution was then centrifuged at $4000 \mathrm{rpm}$ and $4{ }^{\circ} \mathrm{C}$ for $2 \mathrm{~h}$. The upper solid layer was collected and rinsed with $30 \mathrm{~mL}$ purified water. The solution was then agitated at $65{ }^{\circ} \mathrm{C}$ for $1 \mathrm{~h}$ and followed by ultra-sonification for $10 \mathrm{~min}$ to disperse the polyethylene particles. The above solution was then added $0.90 \mathrm{~g} / \mathrm{cm}^{3}$ and $0.96 \mathrm{~g} /$ $\mathrm{cm}^{3}$ isopropyl alcohol solutions with an amount of $2 \mathrm{~mL}$ respectively and followed by the centrifugation at $4000 \mathrm{rpm}$ and $25^{\circ} \mathrm{C}$ for $1 \mathrm{~h}$. The particles existing in the interface between two different isopropyl alcohol solutions were collected. Particles that were dispersed well in a known volume of the solution were collected on a $0.1 \mu \mathrm{m}$ pore size membrane through a vacuum filtration process. The particles collected on the filter paper were examined by using a scanning electron microscope. Micrographs of the particles were then analyzed by using the image analyzer software (Scion Image, a PC version of NIH Image) to measure their dimensions. Measurements were made for at least

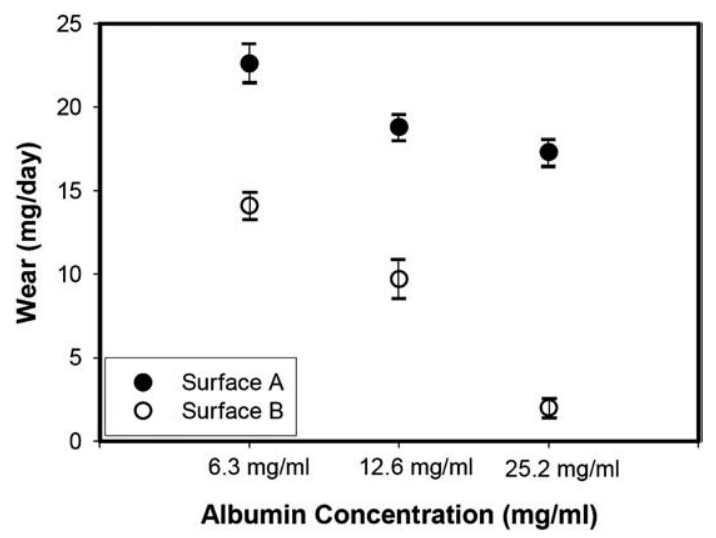

Fig. 4. Wear of UHMWPE in albumin solutions with different concentrations. 
(a)

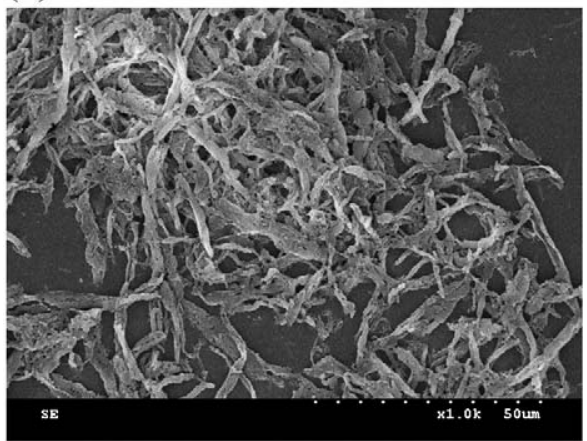

(b)

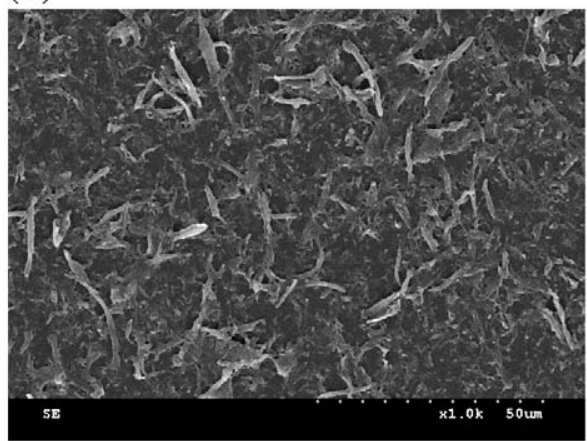

(c)

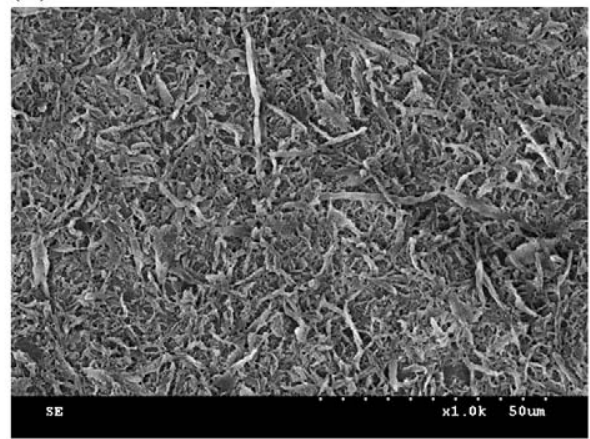

(d)

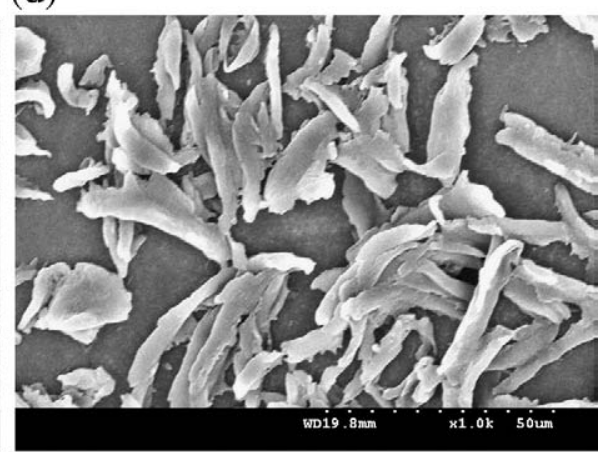

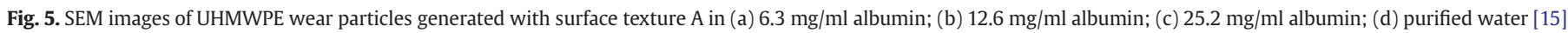

300 particles in each case. The chemical composition of collected particles has been confirmed to be polyethylene by FTIR measurements (Nicolet) from our previous study [16].

\subsection{Protein structure analysis}

The conformation of albumin in solution was monitored using a circular dichroism spectroscopy (CD, Spectropolarimeter J-810, Jasco). $\mathrm{CD}$ is particularly well suited to determine the $\alpha$-helix content of proteins in solution. The wavelengths of $208 \mathrm{~nm}$ and $222 \mathrm{~nm}$ are the sensitive indicators of $\alpha$-helix content. As the protein denatures, the ellipticity of the $\alpha$-helical domains decreases toward to zero. The conformations of albumin molecules before and after wear tests were compared. To obtain the optimal signal conditions, the concentration of albumin solution had to be reduced to $1 \mathrm{mg} / \mathrm{ml}$. The sample solution was about $400-500 \mu \mathrm{l}$ to filled into the cuvette.

\subsection{Contact angle measurement}

Contact angles of albumin solution $(12.6 \mathrm{mg} / \mathrm{ml})$ before and after wear tests on the UHMWPE and Si surfaces were measured by a FTA1000 (First Ten Ångstroms, USA) contact angle and surface tension instrument. Before the measurement, UHMWPE and Si surfaces were ultrasonic cleaned in a 70\% ethanol solution bath for 15 min and in the purified water for another $15 \mathrm{~min}$. The samples were then blow-dried by nitrogen gas. For every measurement, $35 \mu \mathrm{l}$ of albumin solution was dropped on the material surface by the micropipette. The image of the droplet on the surface was captured and the contact angle was calculated by the software.

\section{Results}

Surface textures with various dimensions of cutting edges and distances between features were microfabricated. Fig. 3 shows the scanning electron microscopy (SEM) images of two surface textures used in this study. The dimensions of the surface textures are listed in Table 1.
Wear of UHMWPE with surface textures A and B in albumin solutions within $24 \mathrm{~h}$ is shown in Fig. 4. SEM images of wear particles of UHMWPE generated with surface textures under albumin solutions are compared to the UHMWPE wear particles generated in water [19] in Fig. 5. The results exhibited that particles generated in albumin solutions were found to be smaller and more fiber-like than the particles generated in water. Elliptic major length and minor length measured from the image analysis represent the particle length and width in this study. The mean particle length and aspect ratio of wear particles generated in water and in serum by surface $A$ are listed in Table 2 .

The CD spectra of $12.6 \mathrm{mg} / \mathrm{ml}$ albumin solution measured before and after the wear test (surface texture A and B, duration: $24 \mathrm{~h}$ ) are shown in Fig. 6. Fig. 7 further illustrates a CD spectroscopy measured under various periods during the wear tests (surface A, $12.6 \mathrm{mg} / \mathrm{ml}$ albumin solution). It is shown that the peak of $\alpha$-helix secondary structure at $\lambda_{1}=208 \mathrm{~nm}$ and $\lambda_{2}=222 \mathrm{~nm}$ was decreased after the wear process. The results indicated that albumin conformation may loss its secondary structure.

\section{Discussion}

\subsection{Accelerated wear testing protocol}

Joint simulators are commonly applied to evaluate the wear of articulating materials by mimic the biomechanical condition in vivo. Long term (several months to years) tests are needed in order to distinguish the wear rate between different materials. However, in

Table 2

Particle length and aspect ratio of UHMWPE particles generated in water, serum and albumin by surface $\mathrm{A}$

\begin{tabular}{llll}
\hline & In water & In serum & In albumin $(12.6 \mathrm{mg} / \mathrm{ml})$ \\
\hline Particle length & $29.1 \pm 9.1 \mu \mathrm{m}$ & $9.8 \pm 3.1 \mu \mathrm{m}$ & $12.6 \pm 3.6 \mu \mathrm{m}$ \\
Aspect ratio & $3.5 \pm 1.5$ & $4.6 \pm 2.2$ & $6.9 \pm 1.9$ \\
\hline
\end{tabular}




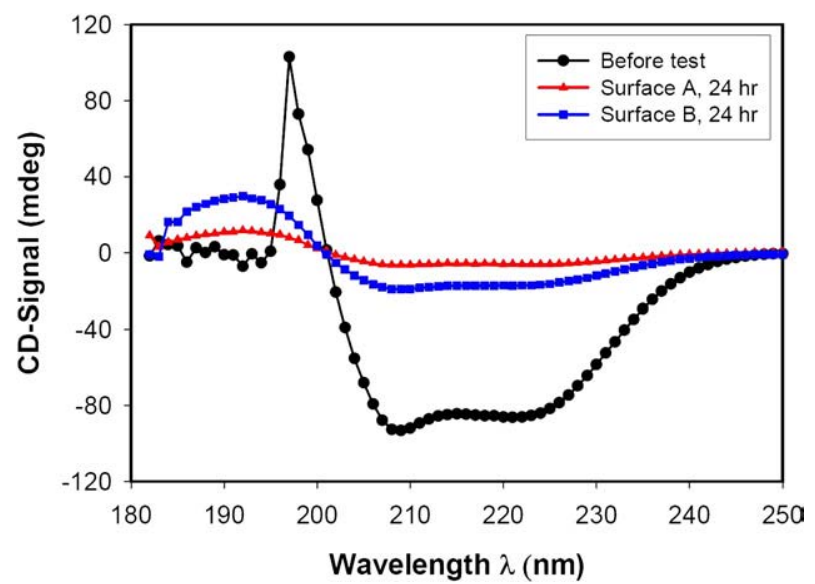

Fig. 6. CD spectra of albumin solutions before and after wear tests.

order to investigate the tribological process induced reaction of the biological lubricants, the long term testing procedure may lead to the evaporation of the solution and the denature of the biological molecules due to the exposure to the environment. We have developed an accelerated wear testing procedure by articulating the joint implant material UHMWPE with microfabricated surface textures. Asperities with controlled dimensions on the material surface can be fabricated through mask design, photolithography and etching process on silicon or metal surfaces [16]. By doing so, the distribution of the contact stress during the articulation process can be defined and controlled. In addition, the microscopic asperity effects on the wear performance of the materials can be measured and discussed in a normal bench testing setup. As seen in Fig. 4, up to $23 \mathrm{mg}$ of UHMWPE wear in $24 \mathrm{~h}$ can be easily quantified. Besides, by further analyzing the morphology of the wear particles, the friction and lubricating ability can be compared. The uniformed surface asperities also make it feasible to perform the theoretical calculation of the contact stress distribution and energy transfer during the lubrication process. In this study, this accelerated wear testing procedure has been successfully employed to investigate the conformational change of albumin induced by friction.

\subsection{Effect of biological lubricant}

Our previous study has indicated that a smaller friction was observed at the interface between the surface feature and UHMWPE in serum than in water [19]. SEM images in Fig. 5 also illustrate that

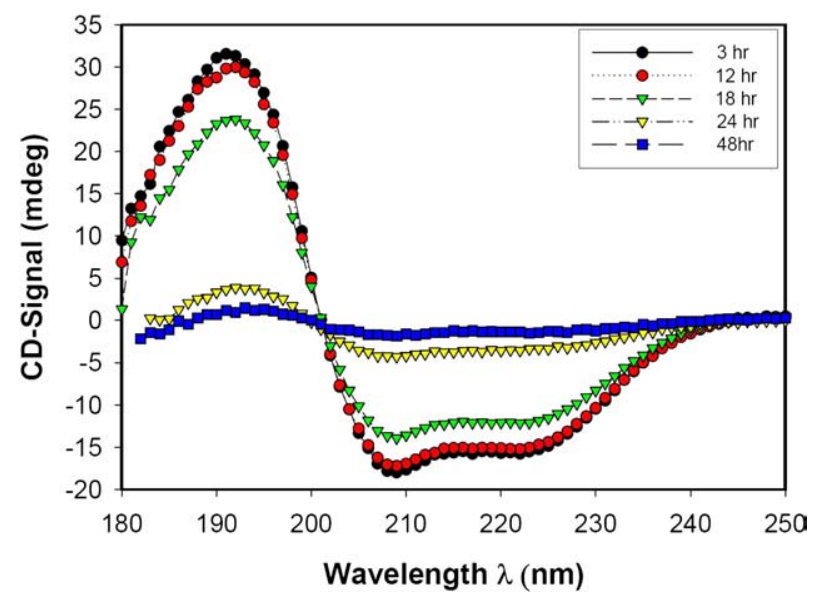

Fig. 7. CD spectra of albumin solutions vs. duration of wear tests. the thinner and fiber-like UHMWPE wear particles were generated in albumin solutions. The length of the particles generated in albumin solution is smaller than the particles generated in water (Table 2). In addition, particle generated in albumin solution has a larger aspect ratio. Our previous study [16] has indicated that the mechanism of the particle generation with microfabricated surfaces consists of the following steps: (a) penetration of the surface feature; (b) Lateral displacement of the feature; (c) Strain hardening of the polymer; (d) Embrittlement of the surface layer by molecular orientation, and (e) Detachment of the particle at the tip edgematerial interface. Thus the friction force between UHMWPE and the surface feature may be reduced with the existence of lubricants. It means a smaller material resistant force to overcome during the sliding process of the surface feature and leads to a smaller lateral displacement of a single surface feature. It is possible that protein molecules existing in albumin solution play significant role to achieve a better lubrication.

It is seen in Fig. 4 that the wear of UHMWPE is decreased with the increasing concentrations of albumin solutions. It is thought that the proteins adsorbed on the material surface acting as boundary lubricating molecules. The lubricating ability of the lubricant depends on the adsorption strength and the coverage area of the molecules on the surface. Once the lubricating molecule was rubbed off in the tribological process, the rate of re-supplying the lubricating molecules to the surface may determines the wear rate of the material. This explains why a higher concentration of albumin solution results in a lower wear of UHMWPE. It is also seen that by using surface A, wear rate of UHMWPE is higher. It may due to the larger "cutting edges" or contact area of the asperities on the articulating surface.

\subsection{Conformational change of albumin}

Fig. 6 compares the CD spectra of albumin solution before and after the wear tests with surfaces A and B. It is shown that the peak of $\alpha$ helix secondary structure at $\lambda_{1}=208 \mathrm{~nm}$ and $\lambda_{2}=222 \mathrm{~nm}$ was decreased after the wear process. It implies that the friction induced the unfolding of the protein structure. During the articulation of the materials, the albumin solution acting as lubricant was squeezed between the materials. Energy dissipated from the friction force may transfer through the lubricant molecules and induces the chemical reaction or conformational change of albumin. It is seen in Fig. 7 that the $\mathrm{CD}$ signals representing the content of $\alpha$-helix decrease with increasing time of the wear process. It is also seen in Fig. 6 that surface A (with larger surface feature length) resulted in a larger amount of loss of $\alpha$-helix content than the surface B did. Those results illustrate that the dissipated energy from friction may correspond to the degree of conformational change of albumin.

Liao et al. [21] has studied the thermal effects on the serum protein contents and Heuberger et al. [22] has discussed the modification of the albumin conformation due to the tribological thermal effect. The adsorption of the related proteins on articulating surface has been investigated and measured $[22,23]$. In addition, Tanaka et al. provided evidence of the pressure-induced denature of human serum albumin [24]. Based on the previous reports and our experimental findings, we suggest that frictional heat and shear stress dissipated energy are probably two major forms of energy causing the decrease of $\alpha$-helix content of albumin.

Table 3

Contact angles of albumin solution $(12.6 \mathrm{mg} / \mathrm{ml}$ ) before and after the wear tests (surface A, $24 \mathrm{~h})$

\begin{tabular}{lll}
\hline & On UHMWPE & On silicon wafer \\
\hline Before wear tests & $79^{\circ}$ & $80^{\circ}$ \\
After wear tests & $73^{\circ}$ & $74^{\circ}$ \\
\hline
\end{tabular}




\subsection{Wear rate issues}

Heuberger et al. had studied the adsorption of human serum albumin on the articulating surface that may affect the friction force [22]. The denatured protein readily adsorbed onto the hydrophobic surface and thus caused the increase of friction force in a pin-on-disc tribometer. However, there exists no observation of the effect of albumin conformational change on the wear of UHMWPE in the previous studies. In this study, we are able to measure the wear of UHMWPE and monitor the conformational change of the albumin. We compared the wear rate of UHMWPE in the $12.6 \mathrm{mg} / \mathrm{ml}$ albumin solution with surface texture $A$. It is found that the wear rate for the first hour is $18.5 \mathrm{mg} / \mathrm{h}$ which is larger than the wear rate of $7.2 \mathrm{mg} / \mathrm{h}$ for the second hour. Decrease of albumin $\alpha$-helix content may contribute to the decrease of UHMWPE wear rate. It is suggested that how the unfolding albumin molecules adhere on the articulating surfaces may provide explanations. Thus, the contact angles of albumin solution $(12.6 \mathrm{mg} / \mathrm{ml})$ before and after wear tests on the UHMWPE and Si surfaces were measured. It is seen in Table 3 that the contact angles of albumin solution after the wear tests become smaller. It implies a better wetting ability or coverage area of the molecule on the articulating surfaces. Combined with the $C D$ measurements, it indicates that an articulating process leads to unfolding of albumin molecules and the unfolding albumin shows a better lubricating ability in this study. To further evaluate the role of the friction induced conformational change of protein in the lubrication of artificial joints in vivo, we need to further investigate the tribochemical kinetics, fluid transportation, and adsorption process of lubricating molecules on the articulating surfaces in the future studies.

\section{Conclusions}

To sum up, the connection of the wear process-induced conformational change of albumin and wear of UHMWPE has been indicated. We have successfully developed and applied an accelerated wear procedure to perform the wear tests. By doing so, the results of wear rate and wear particle morphology of UHMWPE provided us information to compare the lubricating ability of various lubricants.
Decrease of $\alpha$-helix content of albumin during the wear process has been characterized in this study. The results indicated that the conformational change of albumin may link to the decrease of UHMWPE wear rate. Decrease of the contact angles on the articulating surfaces also provided a possible explanation. We have identified the relationship among the tribological process, lubricating molecule structure and wear of UHMWPE.

\section{Acknowledgement}

The authors are pleased to acknowledge the financial support from National Taipei University of Technology (NTUT 97-140-3).

\section{References}

[1] T.M. Wright, S.B. Goodman, Am. Acad. Orthop. Surg. (2001) 3.

[2] D. Dowson, Proc. Inst. Mech. Eng. 205 (1991) 1.

[3] E.J. Cheal, M. Spector, W.J. Hayes, J. Orthop. Res. 10 (1992) 405.

[4] S.L. Evans, P.J. Gregson, J. Mater. Sci., Mater. Med. 5 (1994) 507.

[5] A. Toni, B. McNamara, M. Viceconti, A. Sudanese, F. Baruffaldi, A. Giunti, J. Mater. Sci., Mater. Med. 7 (1996) 149.

[6] H. Weinans, R. Huiskes, J. Biomech. Eng. 116 (1994) 393.

[7] S.M. Kurtz, O.K. Muratoglu, M. Evans, A.A. Edidin, Biomaterials 20 (1999) 1659.

[8] H. McKellop, F.W. Shen, B. Lu, P. Campbell, R. Salovey, J. Orthop. Res. 17 (1999) 157.

[9] O.K. Muratoglu, C.R. Bragdon, D.O. O'Connor, M. Jasty, W.H. Harris, J. Arthroplast. 16 (2001) 149.

[10] R.S. Laskin, J. Bone Jt. Surg. Am. 60 (1978) 182.

[11] L. Marmor, J. Bone Jt. Surg. Am. 61 (1979) 347.

[12] K.A. Elsaid, G.D. Jay, Arthritis Rheum. 52 (2005) 1746.

[13] R.A. Gatter, H.R. Schumacher, A practical handbook of joint fluid analysis/ Philadelphia, Lea\&Febiger press, 1991.

[14] H.-W. Fang, S.M. Hsu, J.V. Sengers, Mater.wiss. Werkst.tech. 34 (2003) 976.

[15] H.-W. Fang, S.M. Hsu, J.V. Sengers, J. Biomed. Mater. Res. B: Appl. Biomater. 67B (2003) 741

[16] H.-W. Fang, S.M. Hsu, J.V. Sengers, NIST SP 1002, , 2003.

[17] S.Y. Yang, W. Ren, Y. Park, A. Sieving, S.M. Hsu, S. Nasser, P.H. Wooley, Biomaterials 23 (2002) 3535.

[18] W. Ren, S.Y. Yang, H.W. Fang, S.M. Hsu, P.H. Wooley, Biomaterials 24 (2003) 4819.

[19] H.W. Fang, Y.C. Su, C.H. Huang, C.B. Yang, Mater. Chem. Phys. 95 (2006) 280.

[20] ASTM F732, American Standards of Testing Materials, 1996.

[21] Y.S. Liao, D. McNulty, M. Hanes, Wear 255 (2003) 1051.

[22] M.P. Heuberger, M.R. Widmer, E. Zobeley, R. Glockshuber, N.D. Spencer, Biomaterials 26 (2005) 1165.

[23] T.A. Blanchet, S.L. Peterson, K.D. Rosenberg, ASME J. Tribol. 124 (2002) 20041.

[24] N. Tanaka, H. Nishizawa, S. Kungi, Biochim. Biophys. Acta 1338 (1997) 13. 